\title{
CORREÇÃO DE ERRO, ATENÇÃO E NOTICING: RELEVÂNCIA PARA A SALA DE AULA
}

\author{
Flávia Azeredo ${ }^{1}$ \\ Heliana Ribeiro de Mello ${ }^{2}$
}

\begin{abstract}
Second language acquisition studies have claimed that feedback, in the form of recasts, has a positive impact on learners' $L 2$ development. This study aims to examine the effectiveness of two corrective feedback forms, recasts and models, on Brazilian learners of English acquiring two language structures and the role of focus attention and noticing in this scenario. The present research was conducted with thirty-three students randomly selected to perform two communicative activities in three different sessions. During the activities, the participants received corrective feedback according to the experimental condition they were assigned. The participants were tested by means of grammaticality judgment tests and oral picture descriptions. Along with the language activities, the participants also performed attention control tests and a stimulated recall test. The results suggest that recasts and models were more effective than no feedback. As for the cognitive factors, the results showed a complex scenario. Overall, attention seems to be the most relevant component of the cognitive variables contemplated on this research.
\end{abstract}

Palarvras-chave: Correção de erro, recast, modelos, aspectos cognitivos, foco de atenção e noticing

\footnotetext{
1 Universidade Federal de Minas Gerais - Morgan State University, Baltimore, MD, USA. American University, Washington, DC, USA - The Johns Hopkins University, Baltimore, MD, USA

2 Universidade Federal de Minas Gerais

Organon, Porto Alegre, nº51, julho-dezembro, 2011, p. 193-216
} 


\section{INTRODUÇÃO}

Por muito tempo, acreditou-se que a evidência positiva era o único elemento necessário para a aquisição ${ }^{3}$ e desenvolvimento do sistema linguístico da primeira língua (L1) e da segunda língua (L2) ${ }^{4}$ do aprendiz. A evidência negativa ${ }^{5}$ ou correção de erro (CE) era vista como desnecessária dentro do processo de aprendizagem (SCHACHTER, 1988, 1991). Os precursores em identificar benefícios advindos de diferentes tipos de CE foram os trabalhos em aquisição de segunda língua (SLA) ${ }^{6}$ dedicados a investigar os possíveis efeitos da interação entre falantes nativos (FN) e falantes não-nativos (FnN) da L2 (LONG, INAGAKI e ORTEGA, 1998; MACKEY e PHILP, 1998; DOUGHTY, IZUMI, MACIUKAIE e ZAPATA, 1999). Em trabalhos posteriores, evidenciou-se que a forma mais frequente de CE era a reformulação implícita de formas agramaticais (PANOVA e LYSTER, 2002; LONG, 2006), a qual ficou conhecida como recast. O recast é uma forma implícita de $\mathrm{CE}$ em que a estrutura linguística a ser alterada é provida ao aprendiz e não incitada a partir dele. Recasts são reformulações de estruturas agramaticais que mantêm o significado central da estrutura original (LONG, 2006) e podem ser de origem sintática, semântica, pragmática e fonológica. Veja exemplo 1:

\section{Exemplo 1 - Recast}

FnN: Why he want this house?

FN: Why does he want this house? $\leftarrow$ recast

(PHILP, 1999, p. 92)

3 Agradecemos todos os comentários e sugestões feitas pelos parecerista anônimos da revista Organon. Quaisquer erros ainda presentes neste texto são da responsabilidade das autoras. Este texto não entrará na discussão da distinção feita por alguns autores, Krashen (1981), por exemplo, entre os termos "aquisição" e "aprendizagem", em L1 ou L2. Esses termos serão empregados aqui de modo intercambiável.

4 Neste artigo, os termos "segunda língua" e "língua estrangeira" serão empregados com o mesmo sentido, referindo-se à língua não-nativa que está sendo aprendida.

5 De acordo com Schachter (1991), a terminologia utilizada para a reformulação de estruturas agramaticais produzidas por aprendizes varia de acordo com a área de estudo. No ensino e aprendizado de L2 emprega-se o termo feedback corretivo. Na linguística, aquisição de L1, usa-se evidência negativa. Na psicologia, na área relacionada ao aprendizado de conceitos, tem-se o termo feedback negativo. Neste artigo, o termo feedback será traduzido para correção de erro e carrega o mesmo sentido empregado por Schachter (1991) de reformulação de formas agramaticais.

6 Optou-se por empregar a sigla em inglês SLA referindo-se à aquisição de segunda língua. 
Contrário à característica implícita do recast, a CE explícita converte o foco da interação do significado para a forma. Estudos como o de Tomasello e Herron (1989), comparando as abordagens foco na forma e foco no significado, e o de Lightbown e Spada (1990), investigando os efeitos da CE, mostraram que a forma explícita de CE é menos produtiva quando comparada à forma implícita. Veja exemplo 2:

\section{Exemplo 2 - CE explícita do professor (P) para um aprendiz (A) \\ A: Uh didn't work well(.) it must be rip=ded rip=ded \\ P: so you need a noun now $\leftarrow$ CE explícita \\ A: it must be rip $=$ ded \\ P: it must be a rip off}

(LOEWEN e PHILP, 2006)

Trabalhos mais recentes estão se concentrando em outros aspectos, tais como: a eficácia da CE implícita e explícita em relação às estruturas linguísticas com diferentes níveis de dificuldade e saliência, com diferentes níveis de complexidade (DEKEYSER, 1995; ROBINSON, 1996; N. ELLIS, 2005) e, estão também, tentando buscar respostas nas diferenças individuais, ou melhor, nas habilidades cognitivas dos aprendizes. Trabalhos concentrando-se na CE durante interações comunicativas têm sugerido que fatores relacionados ao desenvolvimento cognitivo do aprendiz, como a atenção e o noticing, podem ter um impacto na eficácia do recast e no desenvolvimento linguístico do aprendiz (ROBINSON, 2002; MACKEY, 2006).

Visando-se a contribuir para o avanço dos estudos da CE implícita na forma de recast, este estudo examina os benefícios do recast em relação à evidência positiva na aquisição de duas estruturas linguísticas da língua inglesa: a ordem de adjetivos e as perguntas indiretas co-encaixadas ${ }^{7}$. Igualmente, para melhor entender o impacto dos fatores cognitivos no processo de aquisição de L2, investiga-se a possível correlação das duas formas de CE com os fatores cognitivos, foco de atenção e noticing.

\section{CORREÇÃO DE ERRO: RECAST E MODELOS}

A CE na aquisição de L2 é uma prática constante nas salas de aula de L2 e mesmo fora destas. Ao longo dos anos, muitos estudos foram de-

7 Tradução das presentes autoras para embedded questions.

Organon, Porto Alegre, no 51, julho-dezembro, 2011, p. 193-216 
dicados a investigar a relevância da CE (LIGHTBOWN, 1983; SCHACHTER, 1984, 1991; LIGHTBOWN e SPADA, 1990), tanto em sua forma oral quanto em sua forma escrita, para o aprendizado e para o desenvolvimento linguístico do aprendiz. Apesar de essa ser uma questão ainda polêmica, progresso tem sido feito na área de SLA em relação ao papel da CE no processo de aprendizagem. Primeiramente, CE pode ser entendida como qualquer indicação passada para o aprendiz de que a produção deste desvia-se a da língua alvo (LA) (LIGHTBOWN e SPADA, 1999). Nesse sentido, pode-se dizer que há duas formas de correção, uma explícita (explicação metalinguística ou clara CE) e outra implícita (confirmações, repetições, recasts, silêncio e até expressões faciais) (SCHACHTER, 1991). Esta também pode apresentar evidência positiva ou evidência negativa. A evidência positiva é um movimento interacional que fornece modelos de estruturas linguísticas possíveis na LA, ou seja, modelos de exemplares linguísticos que estão presentes no discurso (LEEMAN, 2003). Os modelos podem acontecer na forma de perguntas, afirmações ou mesmo instruções que modelam aspectos linguísticos específicos (R. ELLIS e SHEEN, 2006).

Por sua vez, a evidência negativa é uma condição necessária para aquisição e desenvolvimento da L2, especialmente no caso de aprendizes adultos (GASS, 1988; SCHACHTER, 1988). As afirmações de Gass (1988), Schachter (1988) e White (1988) são comprovadas por White (1991) em um estudo investigando os efeitos da instrução focada na forma ${ }^{8}$, incluindo evidência positiva e evidência negativa, com crianças francesas aprendendo inglês. A evidência negativa pode acontecer de forma direta (explícita), por exemplo, explicações metalingüísticas e, também, de forma reacional. Os recasts são formas complexas de CE, porque podem ser administrados de forma parcial ou total, ou seja, como uma reposta a um único erro ou a múltiplos erros do aprendiz (GASS e SELINKER, 2001). Acredita-se que um dos pontos mais relevantes do recast é que este leva o aprendiz a perceber a discrepância entre a sua produção inicial e a produção ideal, o primeiro passo para o aprendiz abandonar a forma errônea e ir em direção à forma ideal (NICHOLAS, LIGHTBOWN e SPADA, 2001). Veja exemplo 3:

8 Tradução das presentes autoras para form-focused instruction. 


\section{Exemplo 3- CE na forma de recast \\ Student: I think a Perth. \\ Teacher: $\quad$ You think it's Perth. $\leftarrow$ recast}

(LONG, 1996, p. 121)

Examinando-se a literatura encontram-se evidências de fatores que podem ter um impacto na eficácia das formas de CE apresentadas. Estes fatores podem ser de natureza linguística como, a saliência, o tipo e a complexidade das estruturas linguísticas (ROBINSON, 1996; GOLDSCHNEIDER e DEKEYSER, 2001; R. ELLIS e SHEEN, 2006) e, também, de natureza cognitiva, por exemplo, a motivação, o noticing e outros (MACKEY e PHILP, 1998; LEEMAN, 2003). De acordo com a literatura existe a necessidade de mais estudos contemplando tais fatores, para melhor entender os benefícios advindos de formas de CE como o recast e modelo (CHAUDRON, 1987; CARROLL e SWAIN, 1993 e MACKEY, 2006).

\section{ASPECTOS COGNITIVOS: FOCO DE ATENÇÃO E NOTICING}

De acordo com o Leow (1998), não existe aprendizado sem atenção, essa afirmação pode ser aplicada a qualquer área dentro do processo de aquisição de L2. Assim, a atenção é elemento necessário para que o subsequente armazenamento da informação da L2, na memória de longo prazo, aconteça e, é também essencial para a formação e verificação de hipóteses em relação à LA. Além disso, a atenção controla o nível de ativação do insumo na memória de trabalho, ou seja, sem a atenção, a informação desapareceria da memória em poucos segundos (SHARWOOD SMITH, 1991; TOMLIN e VILLA, 1994; SCHMIDT, 2001; LEOW e BOWLES, 2005; ORTEGA, 2009).

Os estudos dedicados à atenção e outros mecanismos de natureza psicolinguística em aquisição de L2 se interconectam com os pressupostos de noticing de Schmidt (1990). A premissa básica da Hipótese de Noticing de Schmidt é a de que o aprendiz precisa notar, conscientemente, a estrutura linguística da L2 no insumo para que a aquisição de tal estrutura aconteça, para que esta se torne intake ${ }^{9}$ (SCHMIDT e

9 Intake foi definido por Schmidt (1990, p. 141) como parte do insumo que os aprendizes percebem ... "se o aprendiz percebe a estrutura linguística no insumo porque este estava, deliberadamente, prestando atenção na forma, ou mesmo se a percepção foi, puramente, não intencional. Quando percebido, o insumo se torna intake”.

Organon, Porto Alegre, nํ51, julho-dezembro, 2011, p. 193-216 
FROTA, 1986; SCHMIDT, 1990, 1993). Para Schmidt (1990), a aprendizagem se dá em um processo consciente e pode ser dividido em três níveis distintos de consciência (awareness): percepção (perception), noticing, compreensão (understanding). Entende-se por percepção (perception), a organização mental e a habilidade do indivíduo de criar representações internas de eventos externos. O segundo, noticing, pode ser entendido como o registro consciente de um evento. O momento em que a informação presente no insumo é apreendida, ao ponto de ser possível fazer um relato verbal. Por fim, compreensão (understanding) é definida como o reconhecimento dos princípios gerais, das regras e das formas.

Dentre os níveis apresentados, o de maior relevância para a área de aquisição de L2 é o noticing. O "noticing é a condição necessária e suficiente para converter o insumo em intake", no sentido de promover o desenvolvimento linguístico do aprendiz (SCHMIDT, 1990, p. 130). Schmidt (1990) menciona que o aprendizado de uma L2 é um processo que precisa ser consciente, logo, noticing é a "atenção consciente ao insumo". De acordo com o autor, o processo de noticing é uma experiência solitária, um acontecimento restrito ao indivíduo e que pode ser comprovado, a partir de relatos verbais do aprendiz. No entanto, a ausência de um relato verbal não implica a não ocorrência de noticing (SCHMIDT, 1990, p. 132), visto que este pode ser evidenciado de outras formas. Outra possibilidade de, indiretamente, verificar a presença de noticing é através de uptake ${ }^{10}$, a incorporação, pelo aprendiz, da correção do interlocutor em sua produção subsequente (ORTEGA, 2009).

No contexto interacional em que o recast acontece o controle do foco de atenção do aprendiz pode delimitar a quantidade de informação verbal que o aprendiz apreende, determinando se o recast foi registrado e processado (TROFIMOVICH, AMMAR e GATBONTON, 2007). Pela natureza do recast, este exige muito das habilidades cognitivas dos aprendizes, especificamente da atenção (TROFIMOVICH et al., 2007).

10 Pondera-se que nesse estudo o construto uptake está sendo empregado como uma das possíveis maneiras de verificar se as formas de CE foram notadas pelo aprendiz. Salienta-se que outros trabalhos já abordaram o construto com o mesmo propósito, por exemplo, Lyster e Ranta (1997); R. Ellis e Sheen (2006); Long (2007). No entanto, o termo teórico uptake pode ser metodologicamente empregado de outras formas dentro da grande área de SLA.

Organon, Porto Alegre, nº 51, julho-dezembro, 2011,p. 193-216 


\section{METODOLOGIA}

\section{$O$ presente estudo}

Este estudo investiga a relevância da evidência positiva (modelos) e da CE implícito negativa na forma de recast no processo de aprendizagem de L2 e contempla também as variáveis de foco de atenção e noticing, que fazem parte do universo psicológico e cognitivo do aprendiz. Especificamente, visa-se a responder a três perguntas neste estudo:

1) O tipo de evidência, recast e/ou modelo, recebida pelo participante, faz alguma diferença para o aproveitamento destes participantes nos testes linguísticos?

2) Os ganhos encontrados no pós-teste são duradouros, quando comparados com o pós-teste tardio?

3) Existe alguma correlação entre as variáveis cognitivas e o tipo de CE empregada?

Os dados linguísticos foram coletados a partir de instrumentos múltiplos e, por sua vez, para os dados cognitivos de foco de atenção empregou-se o teste de Trial Making, e para os dados de noticing utilizou-se uma entrevista estimulada. Estes foram analisados de forma quantitativa e qualitativa.

\section{Participantes}

Os participantes foram selecionados de forma voluntária e aleatória, em um curso de línguas de Belo Horizonte. Todos eram aprendizes no nível intermediário de inglês, no início do segundo ano de aprendizado formal ou o equivalente a, aproximadamente, 120 horas/aula. Todos eram brasileiros, adultos, com idade variando de 19 a 49 anos, média de 28,76 e desvio padrão de 8,76 . Nenhum dos participantes reportou ter problemas auditivos, de aprendizagem ou deficiência de concentração.

Todos passaram por um teste de habilidade oral na LA e fizeram também um pré-teste, escrito e oral, quanto ao conhecimento das estruturas gramaticais aqui estudadas. Foram incluídos na pesquisa os aprendizes que apresentaram conhecimento mínimo ou nenhum conhecimento prévio das estruturas alvo e habilidade oral satisfatória, ou seja, aqueles com capacidade de construir períodos simples na LA.

Organon, Porto Alegre, nº 51, julho-dezembro, 2011, p. 193-216 


\section{Desenho da pesquisa}

Utilizou-se o robusto modelo pré-teste, tratamento, pós-teste, pósteste tardio. Neste modelo, tanto as estruturas quanto os tratamentos e os testes foram cruzados e contrabalançados. O cruzamento e o contrabalanceamento foram utilizados para minimizar e/ou controlar possíveis efeitos de prática no tratamento (carryover effect), ou seja, para que os participantes não se familiarizassem com o tratamento ou percebessem no que estavam sendo investigados (MACREADY, 2006). Esta manobra é aconselhável para assegurar a validade externa quando os informantes são acessados mais de uma vez.

\section{Estruturas alvo}

As estruturas gramaticais contempladas foram: (1) Ordem de adjetivos - Na língua inglesa, os adjetivos têm que obedecer a uma ordem fixa, de acordo com a sua classificação ${ }^{11}$. Neste estudo, investigou-se a sequência tamanho, forma, cor e/ou tamanho, cor, material, como exemplificado:

\section{Exemplo 4 - Adjetivos: tamanho, cor, material}

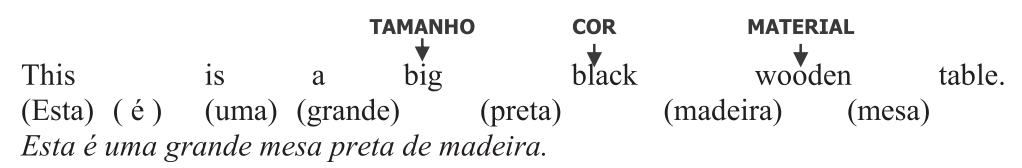

\section{Exemplo 5 - Perguntas no discurso indireto}

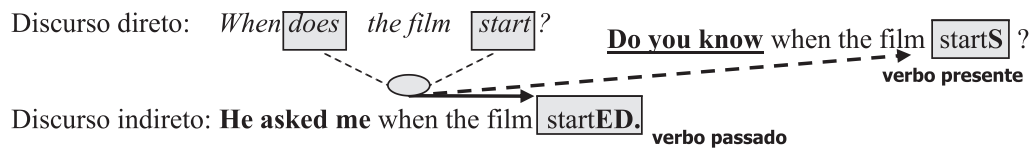

Observa-se, no exemplo 6, que a ordem do sujeito e do verbo não é invertida para a formação destas, como em perguntas regulares.

\footnotetext{
$11 \mathrm{Na}$ L2, expressões socialmente já estabelecidas na língua, por exemplo, This is my old big man, I read a short old story, parecem fugir à regra, no entanto, os adjetivos old e short não são apenas adjetivos, mas modificadores do substantivo. Outro aspecto é o emprego de padrões entoacionais para diferenciar um substantivo composto de um adjetivo seguido de substantivo, por

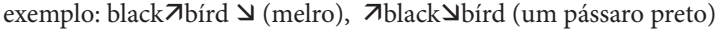

$\rightarrow$

(PRATOR e ROBINETT, 1985). 


\section{Exemplo 6 - Perguntas no discurso indireto}

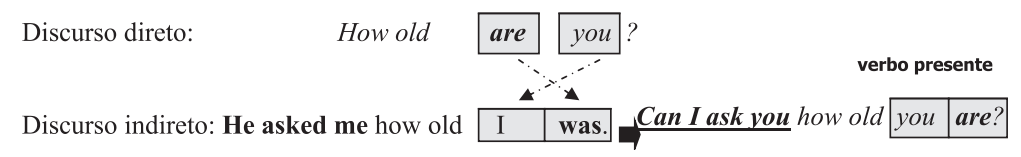

Além das diferenças entre a L1 e a L2, essas estruturas são formas que apresentam baixo valor comunicativo e tendem a não causar interrupções na comunicação. Construções divergentes da forma padrão não interferem na comunicação fazendo com que as imperfeições passem despercebidas pelo aprendiz. Esse fenômeno, de acordo com VanPatten (1996), é um obstáculo para o desenvolvimento linguístico do aprendiz por que o aprendiz não tem a oportunidade de notar as discrepâncias entre a sua produção e a produção ideal.

\section{Instrumentos}

Os dados foram coletados por meio dos instrumentos descritos a seguir: (1) Teste de habilidade oral na LA: este instrumento consta de três conjuntos de figuras, como objetivo de avaliar a capacidade dos participantes de se comunicarem na LA, o nível de vocabulário (tipos de adjetivos e a complexidade de estruturas interrogativas) e, também, se estes tinham algum conhecimento prévio das estruturas que seriam investigadas.

(2) Instrumentos avaliativos para pré-teste, pós-teste e pós-teste tardio: três versões do teste metalinguístico de Julgamento Gramatical (JG). O teste de JG foi elaborado contendo 25 frases, sendo: 10 cobrindo adjetivos, 10 perguntas indiretas e cinco para desviar a atenção dos participantes. As 25 frases foram elaboradas e aleatoriamente selecionadas de um banco de sentenças contendo 90 itens. A atividade foi elaborada seguindo o desenho de testes fechados de múltipla escolha e não foi determinado tempo para os participantes concluírem o teste.

(3a) Três versões do teste de descrição oral de figuras. Elaborou-se um enredo, em que os participantes examinariam uma figura e dariam descrições dessa para um designer gráfico. Em cada uma das atividades tinha-se entre dez e treze slides e, para cada slide, o participante era solicitado a construir oralmente uma ou mais sentenças.

(3b) Três versões do teste para incitar perguntas indiretas co-encaixadas: foram elaboradas três situações diferentes onde os participantes 
tinham que desempenhar o papel de um repórter e fazer perguntas para o interlocutor. Em cada uma das atividades tinha-se entre seis e oito slides e, para cada slide, o participante era solicitado a fazer, no mínimo, duas perguntas.

(4) Tratamentos - Três versões do tratamento para levantar a produção das estruturas da LA: um enredo central foi elaborado em que uma pessoa estava morando na casa de alguns amigos enquanto procurava emprego como jornalista. Finalmente, consegue o emprego, muda-se para Londres, compra móveis para seu apartamento e vai realizar seu primeiro trabalho.

(4a) Ordem de adjetivos: o participante tem que fazer compras de roupas e acessórios para seus amigos, a partir de um catálogo (slides do PowerPoint). Para a condição recast os itens já estavam selecionados. Na condição modelo, o participante vai fazer a mesma compra, no entanto, os amigos deixaram um recado na secretária eletrônica, dizendo o que era para ser comprado. O participante ouve a gravação e efetua as compras. Na condição controle, utilizou-se o mesmo insumo visual da condição recast.

(4b) Perguntas indiretas: o participante vai entrevistar um candidato à vaga de jornalista (condição recast). Na condição modelo, o chefe iria fazer a entrevista com o candidato, mas não pôde. No entanto, o chefe deixou gravadas as perguntas que gostaria que fossem feitas aos candidatos. $\mathrm{O}$ participante precisa ouvir as perguntas e fazê-las aos candidatos. Na condição controle, utilizou-se o mesmo insumo visual da condição recast.

(5) Teste para investigar o controle de foco de atenção (Trial Making Test). O teste foi originalmente desenvolvido em 1944, como parte da Bateria de Testes Individuais do exército americano. Este teste é linguisticamente neutro e se propõe a avaliar a capacidade do indivíduo em variar o foco de atenção entre dois estímulos diferentes. A parte A consiste de uma sequência de números de 1 a 25, distribuídos aleatoriamente em uma folha de papel. O participante precisa ligar os números em ordem crescente, por exemplo, 1-2-3-4-5... 25. Na parte B, têm-se números de 1 a 13 e letras de A-K, também distribuídos aleatoriamente. O participante precisa intercalar números e letras em ordem crescente e alfabética, 1-A-2-B-3-C...13-K. Cada uma das partes da tarefa foi cronometrada, de modo que o resultado da diferença representa a eficácia do indivíduo em alternar seu foco de atenção entre tarefas cognitivas e, também, entre duas sequências de estímulos. 
(6) Entrevista estimulada com o objetivo de investigar a percepção dos participantes quanto aos tratamentos, por exemplo, saber se eles aprenderam algo durante as sessões na semana anterior. Esta foi baseada em Gass e Mackey (2000), as quais utilizaram entrevistas para acessar a eficácia do tratamento, na forma de noticing. No presente estudo, a entrevista foi elaborada de forma que a mesma pergunta fosse reformulada de maneira diferente, com o objetivo de confirmar a resposta do participante ou tentar incitar alguma informação nova a respeito das estruturas, por exemplo, regras gramaticais (APENDICE A).

\section{PROCEDIMENTOS}

Os informantes foram randomicamente distribuídos em seis grupos de sete participantes, os quais foram denominados grupos 1, 2, 3, 4, controle 1 e 2 . Os grupos de tratamento receberam formas de recast e modelos contendo as estruturas alvo, enquanto os grupos controle foram expostos ao mesmo estímulo visual que os demais grupos, mas não receberam nenhum tipo de correção.

No primeiro dia, os participantes fizeram o teste de habilidade oral na LA; logo após, fizeram o pré-teste de JG, de descrição de figuras e de perguntas; por último, foram solicitados a fazer o teste cognitivo de foco de atenção. A realização dessas atividades teve duração de cerca de 30-45 minutos.

O segundo, o terceiro e o quarto dia foram dedicados ao tratamento. Este foi ministrado em três sessões, em três dias consecutivos, com duração de, aproximadamente, 15-20 minutos cada sessão. Em cada sessão, o aprendiz e o interlocutor entraram em uma sala, anteriormente preparada, contendo um computador portátil, um rádio gravador, um microfone unidirecional e um fone de ouvido. $\mathrm{O}$ pesquisador passou, verbalmente, as instruções ao informante, quanto aos procedimentos a serem realizados durante o experimento e lhe passou o material necessário para a realização das tarefas. No decorrer de cada sessão, o interlocutor realizou movimentos de $\mathrm{CE}$, de acordo com o grupo de pesquisa do participante, seguindo um protocolo pré-definido. No quarto dia, após a sessão de tratamento, foi realizado o pós-teste com duração de 15-20 minutos. No quinto dia, ou seja, sete dias depois de encerrado o tratamento, os informantes fizeram o pós-teste tardio - um

Organon, Porto Alegre, nº 51, julho-dezembro, 2011, p. 193-216 
último teste de JG, de descrição de figuras e de perguntas indiretas. Em seguida, os participantes fizeram uma entrevista estimulada para acessar a percepção desses quanto ao experimento. Todas as sessões foram gravadas em áudio e posteriormente transcritas.

\section{RESULTADOS}

\section{Correção de erro: recast e modelo}

O nível de significância estabelecido para as análises foi de 0.05. Em relação ao desempenho dos participantes de acordo com o tipo de CE recebida e o teste aplicado (JG e Oral), observa-se nos gráficos de progressão (FIGURA 1 e 2) que os grupos recast e modelo apresentaram um desempenho superior ao grupo controle. O grupo recast, FIGURA 1, apresentou desenvolvimento maior em relação aos demais grupos. Mesmo que os resultados do grupo recast, no pós-teste, não tenham sido mantidos no pós-teste tardio, os ganhos obtidos por esse grupo ainda são superiores aos dos demais grupos. Os resultados evidenciados no gráfico sugerem que a CE, na forma de recast, parece ter um impacto maior no desenvolvimento linguístico do participante em relação aos resultados dos grupos modelo e controle.

Analisando-se a figura 2, observa-se que os três grupos apresentaram desenvolvimento positivo, comparando o pré-teste com o pós-teste e o pós-teste tardio. Os grupos recast e modelo mostraram maiores ganhos em relação ao controle. Contrariamente à figura 1 , os resultados do grupo recast no pós-teste foram mantidos no pós-teste tardio. Novamente, os resultados do grupo recast, na figura 2, foram superiores aos dos grupos modelo e controle mantendo a mesma tendência encontrada na figura 1.

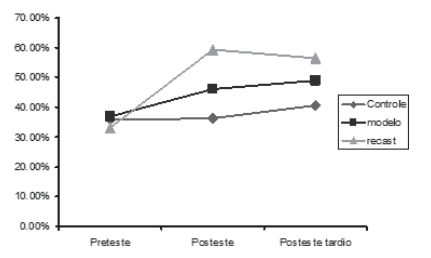

Figura 1: Julgamento gramatical geral

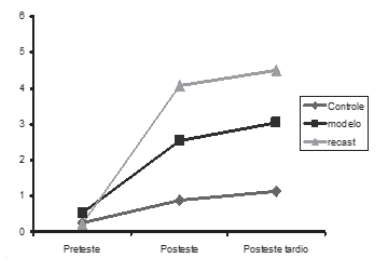

Figura 2: Teste oral geral

Organon, Porto Alegre, nº 51, julho-dezembro, 2011, p. 193-216 
Considerando-se os resultados, separadamente, para o teste de JG para ordem dos adjetivos e perguntas indiretas co-encaixadas, foi encontrado o mesmo padrão da figura 1 de resultados gerais. Os grupos experimentais modelo e recast mostraram ganhos superiores ao controle $(\mathrm{p}<0,05)$ do pré-teste para o pós-teste e do pré-teste para o pós-teste tardio. O grupo recast apresentou uma diferença positiva em relação ao grupo modelo, no entanto, essa diferença não foi estatisticamente significativa $(\mathrm{p}>0,05)$. Observa-se um declínio no aproveitamento do grupo recast do pós-teste para o pós-teste tardio. Os grupos recast e modelo não apresentaram diferença estatisticamente significava entre pós-teste e o pós-teste tardio ( $\mathrm{p}>0,05)$, mas apresentaram diferença estatisticamente significativa em relação ao grupo controle $(\mathrm{p}<0,05)$. Este resultado sugere que, para a ordem dos adjetivos, não há uma diferença significativa entre o emprego de um método ou outro, relativamente, os resultados indicam que ambos os métodos podem levar o aprendiz ao mesmo desenvolvimento.

Nos resultados dos testes de JG para perguntas indiretas co-encaixadas, os grupos modelo e controle apresentaram, proporcionalmente, o mesmo desempenho ao longo do estudo, ou seja, o quadro evidenciado no pré-teste foi mantido para o pós-teste e pós-teste tardio. Por outro lado, constata-se um aproveitamento positivo para o grupo recast em relação aos demais grupos $(\mathrm{p}<0,05)$. Esta diferença é estatisticamente mantida nos dois instantes de coleta de dados, pós-teste e pós-teste tardio. Estes resultados sugerem que, para as perguntas indiretas, apenas a CE na forma de recast parece ser eficaz, quando comparada com os demais grupos.

Nos testes orais para a ordem de adjetivos e estruturas argumentais, podem ser observados os mesmos padrões encontrados na figura 2. Os resultados dos testes orais para as construções com adjetivos, recast e modelo, apresentaram um nível de aproveitamento superior ao do grupo controle $(\mathrm{p}<0,05)$. Especialmente para a ordem dos adjetivos, o grupo modelo apresentou desempenho comparável ao do grupo recast, no entanto, em valores absolutos, o grupo recast teve uma pequena vantagem em relação ao modelo, porém, essa não é estatisticamente significativa $(\mathrm{p}>0,05)$. Para as perguntas indiretas, o grupo recast mostrou ganhos destacadamente superiores aos dos grupos modelo e controle, os quais ficaram relativamente inalterados $(\mathrm{p}<0,05)$. Estes resultados sugerem

Organon, Porto Alegre, nº 51, julho-dezembro, 2011, p. 193-216 
que, para a ordem de adjetivos, os dois métodos, modelo e recast, são semelhantes, no entanto, para as perguntas indiretas o recast é superior.

De forma geral, os resultados encontrados sugerem que a CE implícita na forma de recast parece ser mais eficaz quando comparada ao modelo e ao controle. Do mesmo modo, os dados sugerem um aproveitamento positivo para o recast em relação a ambas as estruturas investigadas, porém, este é mais acentuado para as perguntas indiretas.

\section{Os testes cognitivos e os tipos de correção de erro}

Foram realizadas diversas análises para avaliar a existência de correlação entre os resultados dos testes linguísticos e foco de atenção, no entanto, estes não se mostraram estatisticamente significativos para a correlação ( $p>0,05)$. Apesar disso, observou-se que o coeficiente de correlação, na maioria das vezes, apresentou um valor negativo. Esta tendência indica uma possível correlação inversa entre a atenção e o aproveitamento nos testes linguísticos, ou seja, quanto menor a diferença entre os testes de foco de atenção (Trial A e B) ${ }^{12}$ maior o aproveitamento nos testes linguísticos.

Analisando-se os escores $\mathrm{Z}$ de foco de atenção e os resultados linguísticos observou-se para o grupo recast-JG e Oral que o grupo 1, acima da média, apresentou desempenho maior em relação aos grupos $2 \mathrm{e}$ 3 , para ambos os testes. O grupo 2, considerado normal, obteve ganhos maiores que o grupo 3. Esse padrão foi consistente para ambos os testes, logo, estes resultados sugerem que o nível de atenção corresponde positivamente com o aproveitamento nos testes linguísticos para a forma de CE implícita recast, embora não se possa discutir significância estatística. A mesma análise foi realizada para o grupo modelo. Os participantes com valores acima da média apresentaram altos valores de progressão quando comparados com aqueles do grupo 2. No entanto, os indivíduos com atenção abaixo da média apresentam um desempenho relativamente alto, especialmente para as tarefas orais. Este resultado ocorreu devido a um único indivíduo apresentar progressão alta em relação aos demais membros do grupo.

Em relação à variável noticing, os resultados encontrados não apresentam evidência de correlação entre a entrevista estimulada e os tes-

12 A análise de correlação entre B e A apresentou uma correlação moderada entre as duas partes do teste, como pode ser evidenciado por $r=0,606$ e pelo valor de $p=0,000$. Este resultado indica que os tempos obtidos em A e B são coerentes. 
tes linguísticos $p>0,005$. Resultados similares foram encontrados por Trofimovich et al. (2007). Em Trofimovich et al. (2007) noticing não correlacionou com nenhuma das variáveis preditoras determinadas no estudo: memória fonológica, memória de trabalho, controle de foco de atenção e habilidade analítica.

No entanto, no caso do presente estudo, analisando os participantes individualmente, foi constatado um aproveitamento maior para os participantes que apresentaram um nível alto de foco de atenção e apresentaram evidência de noticing, especialmente para o grupo recast, em ambos os testes. Como mencionado, os resultados não apresentaram o suporte da significância estatística.

\section{DISCUSSÃO DOS RESULTADOS}

\section{Correção de erro: recast e modelo}

Retomando-se a pergunta de pesquisa número 1, em relação ao papel das formas de CE, recast e modelo, para o aprendizado de duas estruturas linguística da L2, pode-se afirmar que o tipo de evidência recebida pelo aprendiz faz diferença. Nesse sentido, a hipótese é parcialmente verdadeira, visto que, quando comparados os efeitos do recast com os do grupo controle, o recast provou ser eficaz para ambas as estruturas, em ambos os testes. Por sua vez, quando se contrasta com o grupo modelo, o recast apresenta um desempenho superior para ambas as estruturas, porém o resultado é estatisticamente significativo, apenas, para as perguntas indiretas, em ambos os testes. Considerando-se a pergunta de número 2, as evidências indicam que sim, já que não foi encontrada uma diferença estatisticamente significativa entre os resultados do pós-teste e do pós-teste tardio, para os grupos recast e modelo. Especificamente, os resultados são positivos para a ordem dos adjetivos, visto que os ganhos entre o recast e o modelo não apresentam uma diferença estatisticamente significativa.

Os resultados deste trabalho sugerem que o tipo de CE recebida pelo aprendiz faz diferença para a incorporação da estrutura em seu sistema linguístico. As evidências encontradas neste estudo são similares às relatadas em trabalhos que igualmente apontaram dados positivos para o recast focado, o qual se concentra em uma única estrutura (LONG, INAGAKI e ORTEGA, 1998; MACKEY e PHILP, 1998; DOUGHTY, 
IZUMI, MACIUKAIE e ZAPATA, 1999 e LEEMAN, 2003). Os resultados de Long et al. (1998 são bastante similares aos do presente estudo. Os autores evidenciaram que os grupos experimentais foram superiores ao grupo controle, sendo que o recast foi mais eficaz para o posicionamento de advérbios. No entanto, nenhum resultado foi encontrado para os pronomes clíticos (lo-la). Este resultado é justificado pelos autores como sendo um problema em relação ao nível de dificuldade da estrutura dado a proficiência da amostra. Segundo Long et al. (1998), corroborado por Goldschneider e DeKeyser (2001), por diversas razões os advérbios são mais salientes, o que faz a estrutura ficar ainda mais perceptível no insumo, o que não é o caso dos pronomes lo-la.

Acredita-se que o cenário descrito em Long et al. (1998) pode ser uma possível explicação para o desempenho dos participantes em relação aos adjetivos no presente estudo. No caso de Long et al., nenhum grupo demonstrou ter aprendido os clíticos. No presente estudo encontrou-se uma situação inversa, pois ambos os grupos parecem ter aprendido a ordem dos adjetivos. Embora as duas estruturas escolhidas para este estudo apresentassem um nível de dificuldade comparável, parece que a ordem dos adjetivos foi menos complexa para os participantes do que a formação de perguntas indiretas. Os adjetivos por si só são caracterizados como sendo estruturas salientes, mas a ordem destes na sentença não é. Porém, ficou mais evidente para os participantes a aquisição do padrão da ordem dos adjetivos, ou seja, a aquisição e ordenação de itens lexicais, do que a aquisição da sintaxe das perguntas indiretas.

Outra possível explicação para o aproveitamento dos participantes, em relação à ordem dos adjetivos é o processo descrito por $\mathrm{N}$. Ellis (2005) como chunking. Este pode ser entendido como um processo que facilita a assimilação de estruturas. À medida que o aprendiz é exposto a uma sequência de exemplares linguístico, a partir da prática, esta sequência é internalizada e mantida na memória. Os subsequentes usos da sequência adicionam velocidade ao processo, ou seja, esta é recuperada da memória e, a partir do uso é automatizada. No caso do presente estudo, não se pode afirmar que a sequência de adjetivos foi automatizada, dado ao curto tempo do experimento, no entanto, o aprendizado a partir da memorização de uma sequência é uma questão a ser considerada.

Organon, Porto Alegre, nº 51, julho-dezembro, 2011, p. 193-216 
Os resultados para as perguntas indiretas parecem ser coerentes, com evidências encontradas na literatura que postulam que os efeitos dos métodos implícitos são favorecidos por estudos de longa duração, com estruturas mais complexas e acessadas por meio de fala espontânea (ROBINSON, 1996 e LONG, 2006). Embora o presente estudo tenha sido de curta duração, o nível de complexidade da estrutura é alto e os dados foram coletados a partir da interação comunicativa, com fala espontânea. Acredita-se que um estudo longitudinal poderia comprovar a durabilidade dos efeitos do recast, visto que esta é ainda uma questão de debate entre os pesquisadores em relação ao recast e aos métodos explícitos. Conforme Dekeyser (1995), os métodos explícitos tendem a favorecer formas gramaticais simples, o que para Robinson (1996) é possível tanto para estruturas simples quanto para complexas, porém, para que isso aconteça, as regras destas estruturas devem ser salientes, o que não é o caso das perguntas indiretas no presente trabalho, logo, uma possível justificativa para o recast ter sido mais eficaz.

\section{Os testes cognitivos e os tipos de correção de erro}

Conforme análise dos resultados encontrados, abordando-se a pergunta de número 3, afirma-se que não foi evidenciada correlação estatisticamente significativa. No entanto, observou-se que os indivíduos os quais apresentaram um alto foco de atenção demonstraram uma maior percepção em termos de noticing das estruturas linguísticas, pelos resultados da entrevista estimulada. Esta tendência se destaca, principalmente, para os participantes que receberam recast. Estes resultados, em parte, podem ser justificados pelo instrumento empregado para coletar os dados de noticing. A literatura mostra que o desafio maior, quando estudando este construto, é encontrar um instrumento eficaz para coletar as informações (SCHMIDT, 2001; LEOW e BOWLES, 2005). Neste estudo, foi empregado um instrumento pós-tratamento, como são tipicamente empregados nos estudos em SLA (ALANEN, 1995 e ROBINSON, 1997), para verificar a relação entre a percepção do participante durante um experimento e o seu aproveitamento após a exposição ao estímulo. Embora sejam amplamente utilizados em pesquisas, ainda existem questionamentos quanto a sua validade, no sentido da veracidade dos dados coletados.

Uma segunda possível justificativa para a falta de correlação entre estas variáveis é a discutida por Trofimovich et al. (2007) que, da mesma

Organon, Porto Alegre, nº 51, julho-dezembro, 2011, p. 193-216 
forma, não encontraram evidência de correlação em seu estudo. Os autores sugerem que resultados como os do presente estudo apenas mostram que os construtos são separáveis. Como discutido por muitos autores, a atenção é necessária para que o aprendiz perceba as discrepâncias da sua produção em relação ao insumo (SCHMIDT, 2001; GASS e SELINKER, 2001), logo, essas variáveis coexistem e, cada uma, tem sua relevância dentro do processo de aprendizagem de L2, mas em níveis diferentes.

\section{LIMITAÇÕES DO ESTUDO}

Uma das principais limitações foi em relação ao número de participantes. Pesquisas futuras deveriam replicar este trabalho contemplando um número maior de participantes. Assim, com mais indivíduos, testes estatísticos mais robustos podem ser aplicados, por exemplo, quando evidenciada a significância estatística entre variáveis, testes de regressão ou teste R2 podem ser realizados.

Outro ponto é em relação à entrevista para coletar dados de noticing. As formas de tratamento empregadas foram ideais para propiciar noticing dos exemplares linguísticos no insumo. No entanto, a entrevista, realizada após o tratamento, não foi um instrumento eficiente, visto que variáveis como o esquecimento e a imaginação dos participantes podem contaminar os dados.

\section{CONCLUSÕES}

Esta pesquisa investigou os benefícios advindos de duas formas de CE, recast e modelo, para a aquisição de duas estruturas linguísticas da LA. Investigou-se também a relação entre estas formas de CE e as variáveis cognitivas, foco de atenção e noticing.

Conclui-se com os resultados que os benefícios do recast e do modelo são superiores à não utilização de CE. Especificamente, para a CE implícita na forma de recast os benefícios estão relativamente ligados ao nível de dificuldade, de complexidade e à saliência da estrutura linguística, como sugerido em Long et al. (1998); R. Ellis e Sheen (2006). Em relação aos aspectos cognitivos e as formas de CE, uma tendência foi encontrada sugerindo que a atenção é o fator cognitivo de maior relevância dentro do processo de

Organon, Porto Alegre, nº 51, julho-dezembro, 2011, p. 193-216 
aquisição, em relação ao noticing. Acredita-se que este resultado é pertinente, visto que a atenção é um construto que igualmente permeia o noticing (SCHMIDT, 1990) e é um fator extremamente relevante dentro do processo de ensino e aprendizagem de forma geral (LEOW e BOWLES, 2005).

Em síntese, este trabalho foi uma tentativa de contribuir com os avanços dos estudos em SLA, na área de CE implícita na forma de recast e para o entendimento do impacto de variáveis cognitivas de diferenças individuais, dentro do processo de aprendizagem de L2. Acredita-se que, mesmo com as limitações, o objetivo maior desta pesquisa foi cumprido, no entanto, espera-se que, para os avanços da área de ensino e aprendizagem de língua, pesquisas futuras tentem responder às questões que este trabalho deixou em aberto.

\section{BIBLIOGRAFIA}

ALANEN, R. Input enhancement and rule presentation in second language acquisition. In: SCHMIDT, Richard (org). Attention and awareness in foreign language learning, p. 259-302. Honolulu, HI: University of Hawai'i at Manoa, Second Language Teaching and Curriculum Center, 1995.

CARROLL, S.; SWAIN, M. Explicit and implicit negative feedback: an empirical study of the learning of linguistic generalizations. Studies in Second Language Acquisition, v. 15, p. 357-386, 1993.

CHAUDRON, C. The role of error correction in second language teaching. In: DAS, B.K (org). Patterns of Classroom Interaction in Southeast Asia, p. 17-50. Singapore: SEAMEO Regional Language Centre, 1987.

DEKEYSER, R. M. Learning second language grammar rules: An experiment with a miniature linguistic system. Studies in Second Language Acquisition, v. 17, p. 379 - 410, 1995.

DOUGHTY, C. Fine-tuning of feedback by competent speakers to language learners. In: ALATIS, J. E. (org). Georgetown university round table on languages and linguistics, p. 96-108. Washington, DC: Georgetown University Press, 1994.

Organon, Porto Alegre, nº 51, julho-dezembro, 2011, p. 193-216 
DOUGHTY, C.; IZUMI, S.; MACIUKAITE, S.; ZAPATA, G. Recasts, focused recasts, and models: effects on L2 Spanish word order. Paper presented at the Second Language Research Forum, University of Minnesota, EUA, 1999.

DOUGHTY, C.; WILLIAMS, J. Issues and terminology. In: DOUGHTY, C.; WILLIAMS, J. (org). Focus on form in classroom second language acquisition, p. 1-11. Cambridge, England: Cambridge University Press, 1998.

ELLIS, N. C. At the interface: dynamic interactions of explicit and implicit language knowledge. Studies in Second Language Acquisition, v. 27, p. 305-352, 2005.

ELLIS, R.; Sheen, Y. Reexamining the role of recasts in second language acquisition. Studies in Second Language Acquisition, v. 28, p. 575-600, 2006.

GASS, S. Integrating research areas: a framework for second language studies. Applied Linguistics, v. 9, p. 198-217, 1988.

GASS, S.; MACKEY, A. Stimulated Recall Methodology in Second Language Research. Mahwah, NJ: Lawrence Erlbaum Associates, 2000. GASS, S.; SELINKER, L. Second Language Acquisition: An Introductory Course. Second Edition. Mahwah, NJ: Lawrence Erlbaum Associates, 2001. GOLDSCHNEIDER, J. M.; DEKEYSER, R. M. Explaining the "natural order of L2 morpheme acquisition" in English: a meta-analysis of multiple determinants. Language Learning, v. 51, p. 1-50, 2001.

ISHIDA, M. Effects of recasts on the acquisition of the aspectual form -te i-(ru) by learners of Japanese as a foreign language. Language Learning, v. 54, p. 311-394, 2004.

IWASHITA, N. Negative feedback and positive evidence in task-based interaction: Differential effects on L2 development. Studies in Second Language Acquisition, v. 25, p. 1-36, 2003.

KRASHEN, S. Second language acquisition and second language learning. Oxford: Pergamon Press, 1981.

LEEMAN, J. Recasts and second language development: beyond negative evidence. Studies in Second Language Acquisition, v. 25, p. 37-63, 2003.

LEOW, R. Toward operationalizing the process of attention in SLA: 
evidence for Tomlin and Villa's (1994) fine-grained analysis of attention. Applied Psycholinguistics v. 19, p. 133-189, 1998.

LEOW, R.; BOWLES, M. Attention and Awareness in SLA. In: SANZ, C. (org). Mind and Context in Adult Second Language Acquisition, p. 179-203. Washington, DC: Georgetown University Press, 2005.

LIGHTBOWN, P. Exploring relationship between developmental and instructional sequences in L2 acquisition In: SELIGER, H; LONG, M. (org). Classroom Oriented Research in Second Language Acquisition Rowley, Mass: Newbury House, 1983.

LIGHTBOWN, P.; SPADA, N. Focus on form and corrective feedback in communicative language teaching: effects on second language learning. Studies in Second Language Acquisition, v. 12, p. 429-448, 1990.

LIGHTBOWN, P.; SPADA, N. How Languages are Learned. Oxford, UK: Oxford University Press, 1999.

LOEWEN, S. Uptake in incidental focus on form in meaning-focused ESL lessons. Language Learning, v. 54, n. 1, 153-187, 2004.

LOEWEN, S.; PHILP, J. Recasts in the adult English L2 classroom: characteristics, explicitness, and effectiveness. Modern Language Journal, v. 90, n. 4, p. 536-556, 2006.

LONG, M. H. Problems in SLA. Mahwah, NJ: Lawrence Erlbaum, 2006.

LONG, M. H. The role of the linguistic environment in second language acquisition. In: RITCHIE, W.; BHATIA, T. (org). Handbook of second language acquisition, p. 413-468. San Diego: Academic Press, 1996.

LONG, M. H.; INAGAKI, S.; ORTEGA, L. The role of implicit negative feedback in SLA: models and recasts in Japanese and Spanish. Modern Language Journal, v. 82, p. 357-371, 1998.

LYSTER, R. Differential effects of prompts and recasts in formfocused instruction. Studies in Second Language Acquisition, v. 26, p. 299-432, 2004.

LYSTER, R.; RANTA, L. Corrective feedback and learner uptake: negotiation of form in communicative classrooms. Studies in Second Language Acquisition, v. 19, p. 37-66, 1997.

Organon, Porto Alegre, n51, julho-dezembro, 2011, p. 193-216 
MACKEY, A.; OLIVER, R. Interactional feedback and children's L2 development, System, v. 30, p. 459-477, 2002.

MACKEY, A.; PHILP, J. Conversational interaction and second language development: recasts, responses and red herrings. Modern Language Journal, v. 82, p. 338-356, 1998.

MACKEY, A.Feedback, noticing and second language development: an empirical study of L2 classroom interaction. Applied Linguistics, v. 27, p. 405-430, 2006.

MACREADY. G. EDMS 645 - Quantitative Research Methods I: Handout Packet. College Park, MD: Copy Service, 2006.

NICHOLAS, H.; LIGHTBOWN, P. M.; SPADA, N. Recasts as feedback to language learners. Language Learning, v. 51, p. 719-758, 2001. ORTEGA, L. Understanding second language acquisition. London: Hodder Arnold, 2009.

PANOVA, I.; LYSTER, R. Patterns of corrective feedback and uptake in an adult ESL classroom. TESOL Quarterly, v. 36, p. 573-595, 2002. PHILP, J. Interaction, noticing and second language acquisition: an examination of learners' noticing of recasts in task-based interaction. Tese (Doutorado). Hobart: University of Tasmania, 1999.

PRATOR, C. H.; ROBINETT, B. W. Manual of American English Pronunciation $4^{\text {th }} e d$. New York: Harcourt College Publishers, 1985.

ROBINSON, P. Effects of individual differences in intelligence, aptitude and working memory on adult incidental SLA: A replication and extension of Reber, Walker and Hernstadt (1991). In: ROBINSON, P. (org). Individual differences in instructed language learning, p. 211-266. Amsterdam/Philadelphia: John Benjamins, 2002.

ROBINSON, P. Individual differences and the fundamental similarity of implicit and explicit adult second language learning. Language Learning, v. 47, n. 1, p. 45-99, 1997.

ROBINSON, P. Learning Simple and Complex Second Language Rules Under Implicit, Incidental, Rule-Search, and Instructed Conditions. Studies in Second Language Acquisition, v. 18, p. 27-67, 1996. SCHACHTER, J. Corrective feedback in historical perspective. Second Language Research, v. 7, p. 89-102, 1991.

SCHACHTER, J. Second language acquisition and its relationship to universal grammar. Applied Linguistic, v. 9, p. 219-235, 1998.

SCHACHTER, J. A universal input condition. In: RUTHERFORD, 
W. (org). Universals and second language acquisition, p. 167-198. Amsterdam, Netherlands: John Benjamins, 1984.

SCHMIDT, R. Attention. In: ROBINSON, P. (org). Cognition and second language instruction, p. 3-32. Cambridge, UK: Cambridge University Press, 2001.

SCHMIDT, R. Awareness and second language acquisition. Annual Review of Applied Linguistics, v. 13, p. 206-226, 1993.

SCHMIDT, R. The role of consciousness in second language learning. Applied Linguistics, v. 11, p. 129 - 158, 1990.

SCHMIDT, R.; FROTA S. N. Developing basic conversational ability in a second language: a case study of an adult learner of Portuguese. In: DAY, R. (org). Talking to Learn: conversation in Second Language Acquisition, p. 237-326. Rowley, MA: Newbury House, 1986.

SHARWOOD SMITH, M. Speaking to many minds: On the relevance of different types of language information for the L2 learner. Second Language Research, v. 7, p. 118-132, 1991.

TOMASELLO, M.; HERRON, C. Feedback for language transfer errors: the Garden Path technique. Studies in Second Language Acquisition, v. 11, p. 385-395, 1989.

TOMLIN, R. S. E VILLA, V. Attention in cognitive science and second language acquisition. Studies in Second Language Acquisition, v. 16, p. 183-203, 1984.

TROFIMOVICH, P. ; AMMAR, A. E GATBONTON, E. How effective are recasts? The role of attention, memory, and analytical ability. In: MACKEY, A. (org). Conversational Interaction in Second Language Acquisition: a series of empirical studies, p. 171-195. Oxford: Oxford University Press, 2007.

VANPATTEN, B. Input processing and grammar instruction in second language acquisition. Westport, CT: Ablex, 1996.

WHITE, L. Adverb-placement in second language acquisition: some effects of positive and negative evidence in the classroom. Second Language Research, v. 7, p. 133-161, 1991.

WHITE, L. Implications of learnability theories for second language learning and teaching. MacGill Working Papers in Linguistics, Cahiers Linguistiques de MacGill, v.5, p. 148-162, 1988.

Organon, Porto Alegre, nº 51, julho-dezembro, 2011, p. 193-216 


\section{APÊNDICE A - ENTREVISTA Pós-tratamento}

Entrevista estimulada: conversa informal com o objetivo de acessar o que os aprendizes notaram durante oxperimento. Os participantes foram solicitados a escrever ou a gravar em áudio

suas respostas.

Nome:

Data:

Por favor, responda:

a) Alguma atividade durante as sessões lhe incomodou?

b) Qual foi sua percepção das atividades? O que você achou das atividades?

c) Você percebeu o que estava sendo estudado nesse trabalho? O que?

d) Você acha que aprendeu alguma coisa nova nessa semana? $\mathrm{O}$ que foi?

e) O que você aprendeu durante o estudo?

f) Sinta-se a vontade para fazer qualquer comentário que você julgue relevante.

Agradeçomos sua colaboração. 